\title{
Physical Study on Motion and Deformation of an Aquaculture Cage Using a 2D and 3D Nonintrusive Imaging Method
}

\author{
Shu-Jing Jan ${ }^{1}$, Ray-Yeng Yang ${ }^{*},{ }^{1}$, Li-An Kuo ${ }^{1}$, Hwung-Hweng Hwung ${ }^{1}$, Chen-Lin Teng ${ }^{2}$ and \\ Hervé Capart ${ }^{2}$ \\ ${ }^{I}$ Tainan Hydraulics Laboratory, Research Center of Ocean Environment and Technology, National Cheng Kung \\ University, Tainan, Taiwan \\ ${ }^{2}$ Civil Engineering Department, National Taiwan University, Taipei, Taiwan
}

\begin{abstract}
The purpose of this study was to solve a fluid-structure coupling problem with the aim of optimizing aquaculture cage design and construction using laboratory observation. In the experiments, the display of a non-intrusive stereo imaging measurement of the three-dimensional net geometry was established by tracing the positions of the LED markers on the cage net. A 1/30-scale model of a net cage was used composing of top and bottom hoops (or rings), nets, four buoys and four anchor blocks moored to the tank bottom. Trinocular stereo imaging, which used three synchronized video cameras to follow the three-dimensional displacements of 37 Light Emitting Diodes (LEDs) attached to the net, was employed to monitor the deformation and motion of the fish net. The motion of the collar and bottom hoop under different current velocities was also observed and is discussed in this paper. This measurement method offers more convenience and detail about deformation and motion investigation of a flexible net structure.
\end{abstract}

Keywords: Net cage, mooring system, non-intrusive stereo imaging method, three-dimensional deformation.

\section{INTRODUCTION}

The requirements for raising seafood have become more and more problematic due to expansion of the global population and decline in the fishery supply. Many efforts to solve this problem in the past have been directed toward marine aquaculture in shallow water.

A major constraint to marine aquaculture development in Taiwan is the lack of suitable water space. Near-shore marine aquaculture still faces the drawback of dispersion of wastes, which limits expansion of the water area of marine aquaculture. To solve the problem, marine aquaculture is moving to the deep sea, which poses some serious problems. The feasibility of extending operations into the energetic open ocean has recently been addressed. However, such a transition is not trivial because suitable species and technologies for this energetic environment have not yet been developed on a viable economic scale.

Recently, a study on near-shore marine aquaculture has brought many advances to light. Bessonneau and Marical [1] developed an interative mathematical method to simulate the dynamic response of selected submerged flexible reticulated surfaces. Lee and Wang [2] studied the dynamic behavior of a tension-leg platform with the net cage system, and also derived a set of equations of motion for a simplified twodimensional case. Based on this, a closed form solution can be obtained when the wave-structure interaction between the

*Address correspondence to this author at the Tainan Hydraulics Laboratory, Research Center of Ocean Environment and Technology, National Cheng Kung University, Tainan, Taiwan;

E-mail: ryyang@mail.ncku.edu.tw top platform structure and the incident wave is considered. Colbourne and Allen [3] measured the wave-induced mooring line forces on a full-scale cage and the wave-induced loads and motions of a model cage. Fredriksson [4] physically and numerically modelled an open ocean fish cage and mooring system dynamics. Aquaculture installations are highly flexible hydroelastic structures, in contrast to most oil and gas facilities. Hydrodynamic forces acting on such a structure affect the shape of the structure, and the altered shape, in turn, affects the hydrodynamic forces. This complex interaction between load and shape is a typical feature of hydroelastic motion. The primary forcing mechanisms due to ocean currents and waves are important in the modelling of open ocean aquaculture systems. Ocean currents induce both normal and tangential steady-state drag forces on the structure components. The nets on the fish cage are often the largest component and have been the focus of drag-force research. Using theoretical and model test methods, Aarsnes et al. [5] determined force and blockage characteristics for individual net types. Lee et al. [6] studied the relationship between the dynamic response of the platform and net-cage features, such as the solidity ratio between the twine diameter and mesh size of the net-cage, while the parameters related to the waves were held constant. In addition, in a structure without a net-cage, the resonant frequency for the netcage system appeared to be reduced as soon as the net was applied, while the amplitude of the response seemed to be increased. However, when the reduction factor was reduced according to the increase of the solidity ratio, both the amplitude and the resonant period were reduced. Furthermore, in an aquaculture application, the internal volume of the net structure influences fish health and well being, and therefore, 
it is important to study how the volume reduction changes due to hydrodynamic force exposure.

The application of digital imaging to trace the fluid or wave is not a new idea. Douxchamp et al. (2002) applied the Voronoï method, which involves positioning and tracking (PTV) of particles dispersed either on the free surface or in the flow fluid, using the 2D method to calculate the results and significant consequence. The capabilities and robustness of these methods are qualitatively illustrated over a wide range of $2 \mathrm{D}$ and $3 \mathrm{D}$ applications. Tsorng et al. [7] studied the three-dimensional tracking of long time trajectories of a suspended particle in lid-driven cavity flow by using a stereo method. In this study, we combine a digital imaging sketch (the non-intrusive imaging method) and experimental measurements to better understand the motion of net cage. Experiments on a 1:30 scale model of a flexible circular net cage with mooring system were conducted in the circulating flume. Collar motion, hydrodynamic force, and net deformation were investigated under different uniform currents. For geometry and motion measurements, a non-intrusive stereo imaging method was employed, using three CCDs with photo frequency of 15 frames per second. The camera systems were used to take the 2D photos, and then the relationship between the 2D imaging coordinates and 3D vision sketches can be obtained by matrix transformation. Furthermore, a submersible load cell was used to investigate the tension force of the anchor systems.

\section{EXPERIMENTAL SETUP AND PHYSICAL MODEL- LING}

This research focuses on a fish net cage system. The interaction between current and the net system alone is under investigation and will be incorporated into the study of the entire cage system in future. A $1 / 30$-scale model of the net cage was composed of a top and bottom hoop (or ring), and nets with a mesh size of $0.6 \mathrm{~cm}$ (net solidity $\mathrm{Sn}=0.33$ ). Four buoys and four anchor blocks were moored to the tank bottom. The top of the net was mounted on the hoop such that it took the form of a cylinder with an open top. The hoop, which utilized buoys to keep the net cage in a location to stably maintain the free surface, was kept in a fixed position during each test. The float collar system, the cage net system, the anchor system, and the mooring system are depicted in Fig. (1).

The experiments were conducted in the circular flume at the Tainan Hydraulics Laboratory (THL), National Cheng Kung University, Taiwan. This tank is a vertical-type circular water channel driven by a screw pump with an observation section of $2.3 \mathrm{~m} \times 1 \mathrm{~m} \times 1.5 \mathrm{~m}$ (length $\times$ height $\times$ width), as shown in Fig. (2). The maximum velocity of the generating uniform flow is $28 \mathrm{~cm} / \mathrm{s}(153.4 \mathrm{~cm} / \mathrm{sec}$ in the prototype).

The components of the physical model and the flow velocity conditions were Froude scaled with the exception of the nets on the fish cage, which utilized Froude scaling with a Reynolds number adjustment technique as described in Palczynski and Fredriksson [8]. The physical model details of the cage and mooring system are shown in Table $\mathbf{1}$.

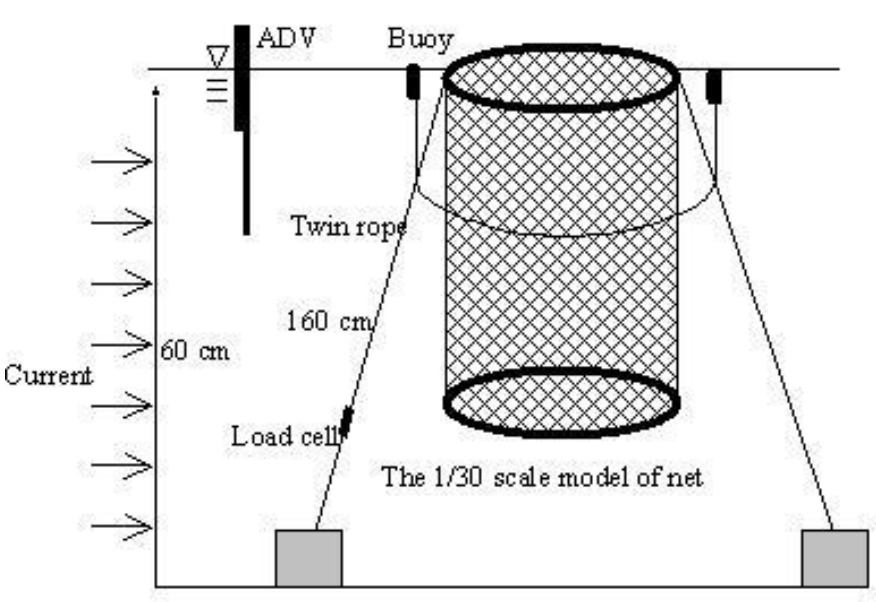

Fig. (1). Configuration of experimental setup at the circular flume tank in the THL.

\section{observation section $(2.3 \mathrm{~m} \times 1 \mathrm{~m} \times 1.5 \mathrm{~m})$}

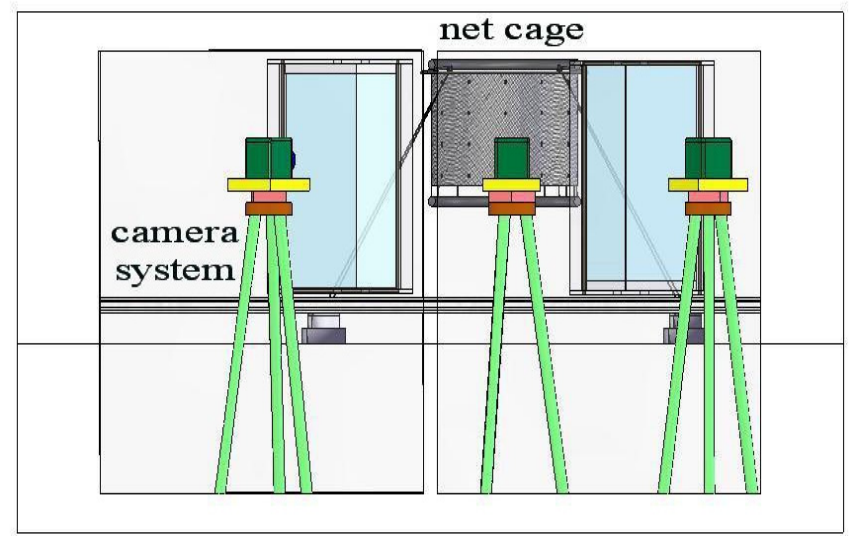

Fig. (2). Observation section $(2.3 \mathrm{~m} \times 1 \mathrm{~m} \times 1.5 \mathrm{~m})$ of the circular flume tank in the THL.

In this study, the experimental setup was used to obtain all net deformation (and motion), collar motion, and mooring tension response to the current action (sampled at a rate of $50 \mathrm{~Hz}$ ). The tension force was measured by one load cell (length $3 \mathrm{~cm}$, diameter $0.5 \mathrm{~cm}$ ) on the mooring cable, with a measurement range and accuracy of $2 \mathrm{~kg}$ and $0.2 \%$, respectively. The net deformation measurements were taken using a non-intrusive stereo method adapted from Spinewine et al. [9] and Douxchamps et al. [10,11]. Furthermore, an estimate of the net geometry was established by measuring several markers on the net. To avoid the effect of deformation and added weight of the net cage, 37 Light Emitting Diodes (LED) were used as position markers. The placement and numbering of the LED markers are shown in Fig. (3). Next, three cameras traced the LED markers with an error range less than $+/-1 \mathrm{~cm}$. The positions of the markers were used to calculate the volume reduction of the net cage. The model was subjected to six different current velocity cases ( $U_{\text {steady }} \approx 6,8,11,14,17$, and $22[\mathrm{~cm} / \mathrm{s}]$ ). Flow speed was measured with an Acoustic Doppler Velocimeter (ADV). 


\section{DESCRIPTION OF 2D AND 3D STEREO IMAGING MEASUREMENTS OF NET DEFORMATION}

In general, the theory of this method could be applied in the 2D and 3D imaging fields. Certain cases measured motion under different conditions, including uniform flow, semi-symmetric, or non-symmetric conditions, which could

Table 1. Net Cage and Mooring System Physical Model Particulars Physical Model Particulars

\begin{tabular}{|c|c|c|c|}
\hline Parameter & \multicolumn{2}{|c|}{ Full-Scale } & Model-Scale \\
\hline \multirow{3}{*}{$\begin{array}{l}\text { Main Rope } \\
\text { Nylon Twist } \\
\text { Rope }\end{array}$} & Diameter & $0.05(\mathrm{~m})$ & $0.2605(\mathrm{~cm})$ \\
\hline & Density & $1,140\left(\mathrm{~kg} / \mathrm{m}^{3}\right)$ & $1.14\left(\mathrm{~g} / \mathrm{cm}^{3}\right)$ \\
\hline & Mass per unit length & $304 / 200(\mathrm{~kg} / \mathrm{m})$ & $0.03879(\mathrm{~g} / \mathrm{cm})$ \\
\hline \multirow{2}{*}{$\begin{array}{l}\text { Bridle } \\
\text { Nylon Twist } \\
\text { Rope }\end{array}$} & Diameter & $0.03(\mathrm{~m})$ & $0.2605(\mathrm{~cm})$ \\
\hline & Mass per unit length & $109 / 200(\mathrm{~kg} / \mathrm{m})$ & $0.03879(\mathrm{~g} / \mathrm{cm})$ \\
\hline \multirow{3}{*}{ Buoy } & Diameter & $0.91(\mathrm{~m})$ & $4.7(\mathrm{~cm})$ \\
\hline & Mass & $41.0(\mathrm{~kg})$ & $5.87(\mathrm{~g})$ \\
\hline & Buoyancy & $750(\mathrm{~kg})$ & $435(\mathrm{~g})$ \\
\hline \multirow{5}{*}{$\begin{array}{l}\text { Collar } \\
\text { HDPE }\end{array}$} & Density & $950\left(\mathrm{~kg} / \mathrm{m}^{3}\right)$ & $0.95\left(\mathrm{~g} / \mathrm{cm}^{3}\right)$ \\
\hline & $\begin{array}{l}\text { Internal circle } \\
\text { length }\end{array}$ & $50(\mathrm{~m})$ & $162(\mathrm{~cm})$ \\
\hline & $\begin{array}{l}\text { Outside circle } \\
\text { length }\end{array}$ & $54(\mathrm{~m})$ & $192(\mathrm{~cm})$ \\
\hline & Diameter & $17.19(\mathrm{~m})$ & $61.1(\mathrm{~cm})$ \\
\hline & Mass & $1,620(\mathrm{~kg})$ & $596(\mathrm{~g})$ \\
\hline \multirow{3}{*}{ Net } & Mesh size & $0.0604(\mathrm{~m})$ & $0.46(\mathrm{~cm})$ \\
\hline & Diameter of line & $0.0021(\mathrm{~m})$ & $0.02(\mathrm{~cm})$ \\
\hline & Depth of net & $10(\mathrm{~m})$ & $30(\mathrm{~cm})$ \\
\hline \multirow{3}{*}{$\begin{array}{l}\text { Bottom hoop } \\
\text { HDPE }\end{array}$} & Density & $950\left(\mathrm{~kg} / \mathrm{m}^{3}\right)$ & $0.95\left(\mathrm{~g} / \mathrm{cm}^{3}\right)$ \\
\hline & Circle length & $54(\mathrm{~m})$ & $162(\mathrm{~cm})$ \\
\hline & Diameter & $17.19(\mathrm{~m})$ & $61.1(\mathrm{~cm})$ \\
\hline
\end{tabular}

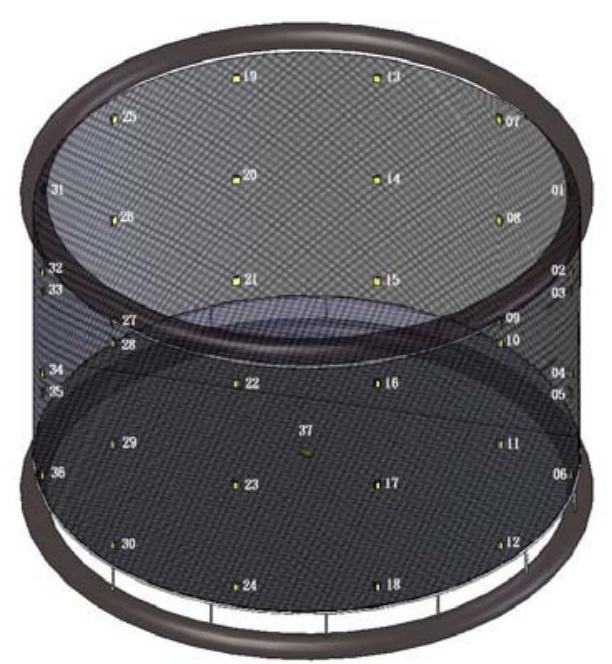

Fig. (3). Placement and numbering of LED markers on the net cage. take advantage of $2 \mathrm{D}$ or $3 \mathrm{D}$ imaging techniques to solve the results. Based on experimental equipment and test conditions, we can use two or more CCD cameras to obtain the images. Thus, the methods and the application of 2D semisymmetric fluid flow or 3D fluid flow will be discussed as follows.

\section{INTRODUCTION OF 2D STEREO IMAGING METHOD}

The basic goal of the stereo imaging method is to find a transformation that relates the set of 2D imaging coordinates of any point $P$ to its 3D coordinates. From Fig. (4), it is assumed that the camera positions at viewpoints $\mathrm{A}$ and $\mathrm{B}$ are known. Then, we can define

$r_{P}=\left[x_{P}, y_{P}, z_{P}\right]^{T}$,

as the $3 \mathrm{D}$ coordinates of point $\mathrm{P}$, and

$R_{p}^{(A)}=\left[X_{p}^{(A)}, Y_{p}^{(A)}\right]^{T}$,

as the 2D image coordinates of point $P$ associated with the camera viewpoint $A$ (point $P^{\prime}$ in left image plane $\Phi$ ). The transformation is performed by modeling the image formation as a central projection from a virtual camera focal point A onto the image plane $\Phi$ (Tasi [12]; Jain et al. [13]). For each viewpoint $A$, one can then specify a matrix $\left[A^{(A)}\right]$ and a vector $b^{(A)}$ such as

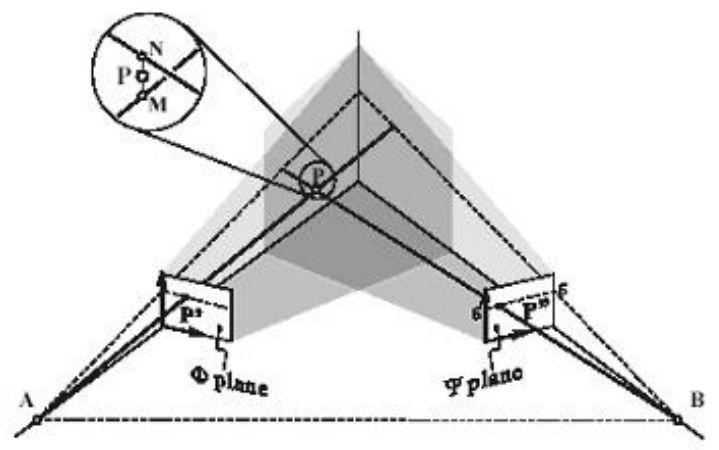

Fig. (4). Imaging geometry: physical point $P$ with its imaging projections $\mathrm{P}^{\prime}$ and $\mathrm{P}^{\prime \prime}$ from the two stereo views.

$\alpha\left[\begin{array}{l}X^{(A)} \\ Y^{(A)} \\ 1\end{array}\right]=\left[A^{(A)}\right]\left[\begin{array}{l}x \\ y \\ z\end{array}\right]+b^{(A)}$

in which $\alpha$ is a scalar parameter interpreted in the context of Eq. 4 below. Matrix $\left[A^{(A)}\right]$ and vector $b^{(A)}$, on the other hand, must be calibrated from a set of points $P_{k}$ for which both the world coordinates $\left[x_{k}, y_{k}, z_{k}\right]^{T}$ and the image coordinates $\left[X_{k}^{(A)}, Y_{k}^{(A)}\right]^{T}$ are known.

Once calibrated, Eq. 3 can be used to obtain the image coordinates from known global coordinates. Conversely, a point $P$ having its projection $P^{\prime}$ with the image coordinates $R_{P}{ }^{(A)}=\left[X_{P}{ }^{(A)}, Y_{P}{ }^{(A)}\right]^{T}$ under viewpoint $A$ is known to belong to a ray $A P$ defined by parametric equation 
$r_{A P}(\alpha)=r_{A}+\alpha S_{A P}$,

$r_{B P}(\alpha)=r_{B}+\beta S_{B P}$,

Let $\mathrm{M}$ and $\mathrm{N}$ be points on both rays separated by the smallest inter-distance. The midpoint of the segment joining $\mathrm{M}$ and $\mathrm{N}$ constitutes an approximation of the true particle position $P$. The length $\ell$ of the segment, on the other hand, measures the distance of the closest encounter between the two rays and provides an estimate of the quality of the approximation. They are given by

$r_{P}=\frac{1}{2}\left[r_{M}+r_{N}\right]$,

$\ell=\sqrt{\left[r_{N}-r_{M}\right]^{T}\left[r_{N}-r_{M}\right]}$,

The position of $\mathrm{M}$ and $\mathrm{N}$ are as follows

$r_{M}=r_{A}+\alpha_{M} S_{A P}$,

$r_{N}=r_{B}+\beta_{N} S_{B P}$,

in which two unknown parameters $\alpha_{M}$ and $\beta_{N}$ are solved by the following linear system

$$
\left(\begin{array}{c}
S_{A P}^{T} S_{A P}-S_{A P}^{T} S_{B P} \\
S_{B P}^{T} S_{A P}-S_{B P}^{T} S_{B P}
\end{array}\right)\left(\begin{array}{c}
\alpha_{M} \\
\beta_{N}
\end{array}\right)=\left(\begin{array}{l}
{\left[r_{B}-r_{A}\right]^{T} S_{A P}} \\
{\left[r_{B}-r_{A}\right]^{T} S_{B P}}
\end{array}\right),
$$

Due to the imperfection of the imaging process, this intersection cannot be exactly determined; therefore, the point of closest encounter between two rays will be refound. Let $\mathrm{M}$ and $\mathrm{N}$ be points on both rays separated by the smallest inter-distance. The midpoint of the segment joining $\mathrm{M}$ and $\mathrm{N}$ constitutes an approximation of the true particle position $P$.

Generally, we can use the imaging method to trace the trajectory of a particle in an experiment. The measurement considers a situation in which a wave or current carrying sand or gravel produces an impact that affects the geometry in the symmetric situation. Therefore, the 2D stereo imaging method is a good technique for application to subjects of symmetric motion.

\section{DEPICTION AND APPLICATION OF THE 3D STE- REO IMAGING METHOD}

We used two cameras to capture the images from the net cage via the side window view. Stereo imaging techniques depend on purely geometrical principles in order to reconstruct the $3 \mathrm{D}$ particle positions, concentrations, and velocities. The method takes advantage of projective geometry and the Voronoi diagram. Ahuja [14] and Okabe et al. [15] illustrated that the core of the proposed method consists of exploiting the special properties of the Voronoi diagrams. A method used to obtain concentration estimates from statistical distributions associated with stereo imaging techniques is described by Murai et al. [16].

Trinocular stereo imaging is used to monitor the deformation of the fish net. For this purpose, three synchronized video cameras are used to follow the three-dimensional displacements of the 37 Light Emitting Diodes (LEDs) attached to the net. The three cameras are placed on one side of the flume, and view the net from straight under (central camera) and oblique viewpoints (left and right cameras). To minimize the optical distortion associated with light refraction across the water-glass-air interfaces of the flume side, the two oblique cameras view the net through two water-filled prisms, following the design proposed by Klar et al. [17].

The transformation from 3D physical coordinates to $2 \mathrm{D}$ image coordinates is modeled as a central projection from a virtual camera focal point onto the image plane. For each of the three cameras, $i=1,2,3$, one can then write the following relationship between coordinates $(x, y, z)=\mathbf{r}$ of a given LED and the corresponding image coordinates $\left(\gamma^{(i)}, \rho^{(i)}\right)=$ (column, row) of the pixel array:

$\lambda^{(i)}\left(\begin{array}{c}\gamma^{(i)} \\ \rho^{(i)} \\ 1\end{array}\right)=\mathbf{A}^{(i)}\left(\begin{array}{l}x \\ y \\ z\end{array}\right)+\mathbf{b}^{(i)}$,

where the 12 coefficients of matrix $\mathbf{A}^{(i)}$ and vector $\mathbf{b}^{(i)}$ are determined using the procedure described in Spinewine et al., based on the known physical and image coordinates of the calibration target. It follows that if a given LED is detected on image $i$ at pixel coordinates $\left(\gamma^{(i)}, \rho^{(i)}\right)$, then its 3D position vector $\mathbf{r}$ can be expressed in parametric form using the ray equation

$\mathbf{r}=\mathbf{p}^{(i)}+\lambda^{(i)} \mathbf{q}^{(i)}$

where $\mathbf{p}^{(i)}=-\left[\mathbf{A}^{(i)}\right]^{-1} \mathbf{b}^{(i)}$ is the projection centre associated with viewpoint $i, \mathbf{q}^{(i)}$ is a direction vector given by

$\mathbf{q}^{(i)}=\left[\mathbf{A}^{(i)}\right]^{-1}\left(\begin{array}{c}\gamma^{(i)} \\ \rho^{(i)} \\ 1\end{array}\right)$,

and $\lambda^{(i)}$ is an unknown parameter denoting the location of the point along the ray. If the point (LED) is seen under two or three viewpoints, then its 3D location will lie at the approximate intersection of 2 or 3 rays. This approximate intersection is found by assembling an over-determined system of linear equations in the unknowns $\lambda^{(i)}$, which can then be solved in the least squares sense. Two viewpoints are actually sufficient to obtain the 3D position of a LED in this fashion, but the advantage of using three cameras is that an LED that may be occluded under one viewpoint is likely to be seen with the other two. Furthermore, if the LED is seen from three viewpoints, its position will be determined more accurately. This is shown in Fig. (5).

To facilitate the determination of fish net deformations, the different LEDs are assumed to constitute the nodes of a deformable mesh structure. The correspondence between the measured LED positions obtained from the trinocular stereo and the nodes of the full mesh is initialized using a semimanual point-and-click procedure, pairing nodes of the actual net in its undeformed state with those of an ideal mesh. The correspondence is then updated for successive stages of 
deformation (for stronger and stronger water currents) using a nearest-neighbor matching scheme.

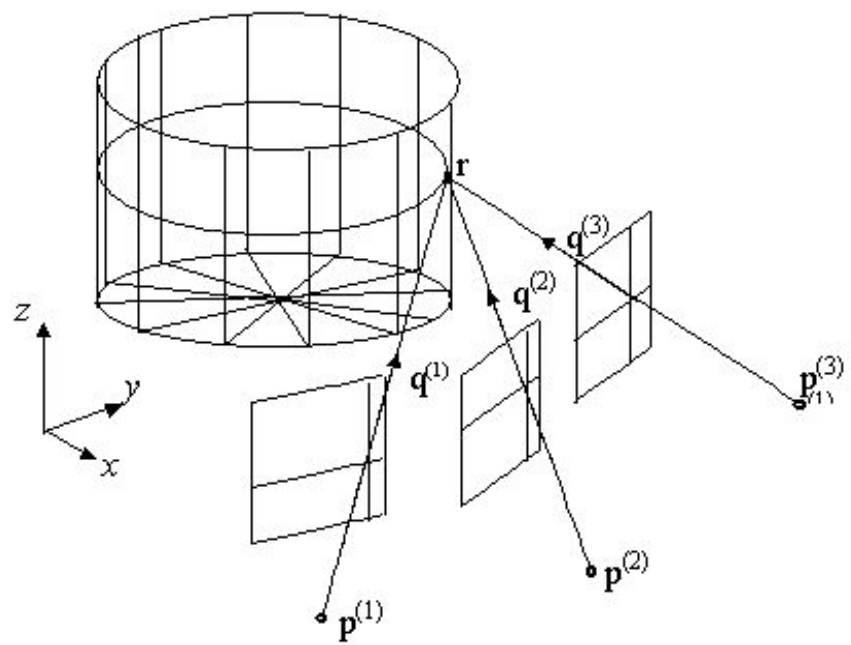

Fig. (5). Trinocular imaging geometry: a given LED is seen under three camera viewpoints and lies at the intersection (in the least squares sense) of three optical rays.

In this study, the above Eqs.1-3 can analyze the positions of LED markers obtained from three CCD camera systems. Then, the motion and the volume reduction can be calculated. Before the experimental test, the correct positions of a calibration target's LED markers (shown in Fig. (6)) in static water were calibrated first. Additionally, in order to minimize the refraction effect for the optical rays passing through a multimedia interface, two prisms filled with water were attached to the observation window.

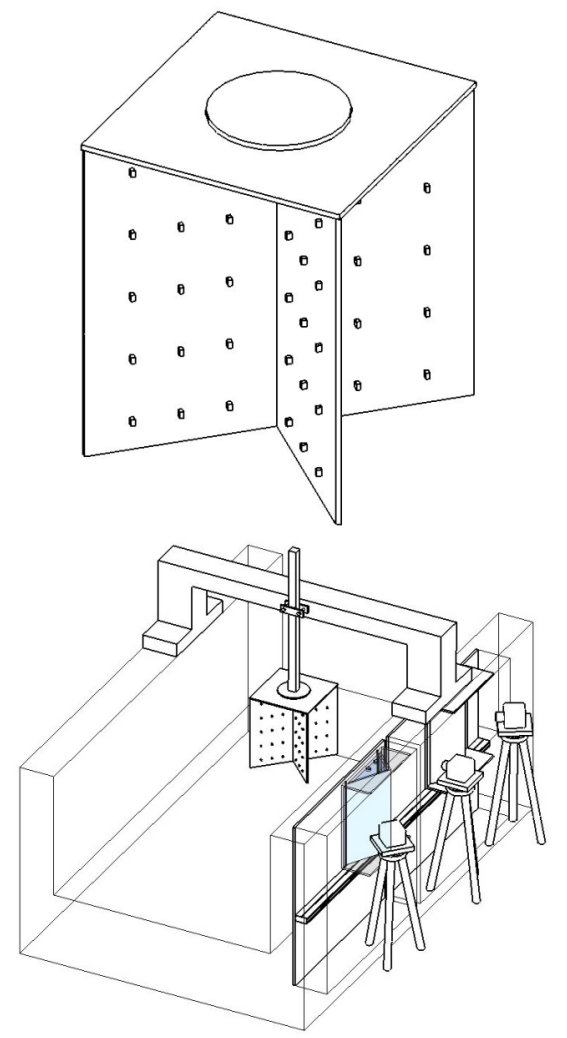

Fig. (6). Configuration of calibration target and side view for the two prisms filled with water.
The $x, y, z$ coordinates of the LED markers are obtained using the $3 \mathrm{D}$ stereo imaging method described above. $\mathrm{A}_{\mathrm{e} 0}$ is the effective exposed area when the net cage is unexposed to current. The exposed area of the mesh is estimated by projecting the measured 3D LED positions onto a transverse plane, and therefore, the area enclosed by the convex hull of the projected positions will be measured. Then, the positions of the LED markers are used to calculate the volume of the net cage by Gaussian divergence theory. We segment the net cage into $n$ triangle elements and use the equation to obtain the volume (V) formula as in the following

$V_{P}=\sum_{i=1}^{n}\left(\vec{f}_{G i} \cdot \vec{n}_{i}\right) d A_{i}$,

in which $\vec{f}_{G i}$ is the location vector passing through the center of gravity of the triangle element; $\vec{n}_{i}$ is the unit normal vector of the triangle element, and $\vec{r}_{i j}$ is the distance between point $i$ and point $j$. This is shown in Fig. (7). $a_{i}, b_{i}$ and $c_{i}$ are the edge lengths of the triangles. Thus,

$\vec{f}_{G i}=\sum_{i=1}^{3}\left((x)_{i} \vec{i}+(y)_{i} \vec{j}+(z)_{i} \vec{k}\right) / 3$,

in which point $G$ is the center of the gravity for the triangle element.

$\vec{n}_{i}=\frac{r_{12} \times r_{23}}{\left|r_{12} \times r_{23}\right|}$,

$d A_{i}=\sqrt{s_{i}\left(s_{i}-a_{i}\right)\left(s_{i}-b_{i}\right)\left(s_{i}-c_{i}\right)}$,

and

$s_{i}=\left(a_{i}+b_{i}+c_{i}\right) / 2$,

The volume deformation rate $\left(C_{v d}\right)$ can be defined as

$C_{v d}=1-\frac{V_{P}}{V_{P o}}$

in which $V_{P o}$ is the volume when the net cage is unexposed to current.

The area of the projection of the net cage onto a plane orthogonal to the incoming current is called the effective exposed area $\left(A_{e}\right)$. Due to the deformation of the net cage, $A_{e}$ is dependent on the current velocity, and decreases with increasing current. When considering the dynamic force on the net cage, the effect of changing $A e$ should be taken into account. Therefore, in order to show the relation of the velocity, projected area and tension forces, the tension force divided by the velocity squared and the exposed area of the deformed cage will be analyzed.

\section{RESULT AND DISCUSSION}

\section{Discussion of the Application of 2D and 3D Imaging Method for Different Velocity Conditions}

In this experiment, in order to evaluate the flow situation, we chose a suitable method to use in analyzing the results. 
Briefly, we applied the 2D method to resolve simple fluid flow or gravel dynamics measurement. This decreases calculation time and saves computer memory space. In non-symmetric flow fluid motion, to trace the trajectory of a particle or the deformation of an object, the 3D method is able to capture the spatial change and shape of the object. This can be used to quantify practical observations and to facilitate analysis of the results in order to understand the impact of current on the net cage or anther object in the experimental system.

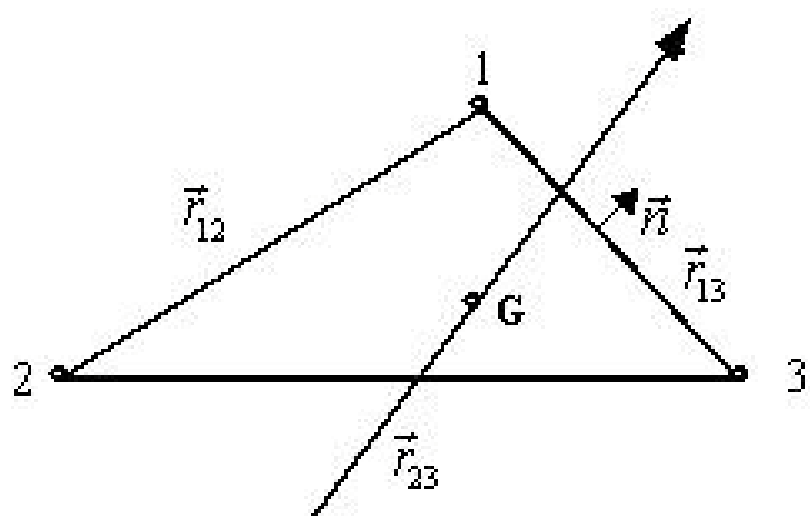

Fig. (7). Triangle element.

\section{Demonstration of Analysis with or without LEDs on Net Cage}

In this experimental study, the stereo imaging method was used to measure volume deformation of the net cage under different current velocities. Additionally, the images were

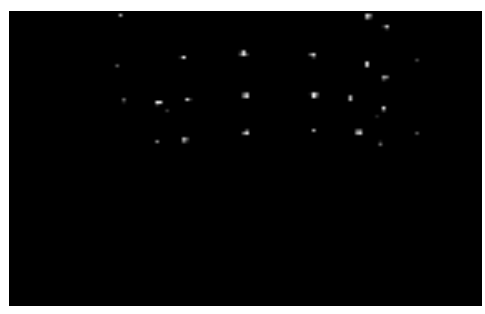

(a) $\mathrm{V}=6(\mathrm{~cm} / \mathrm{s})$

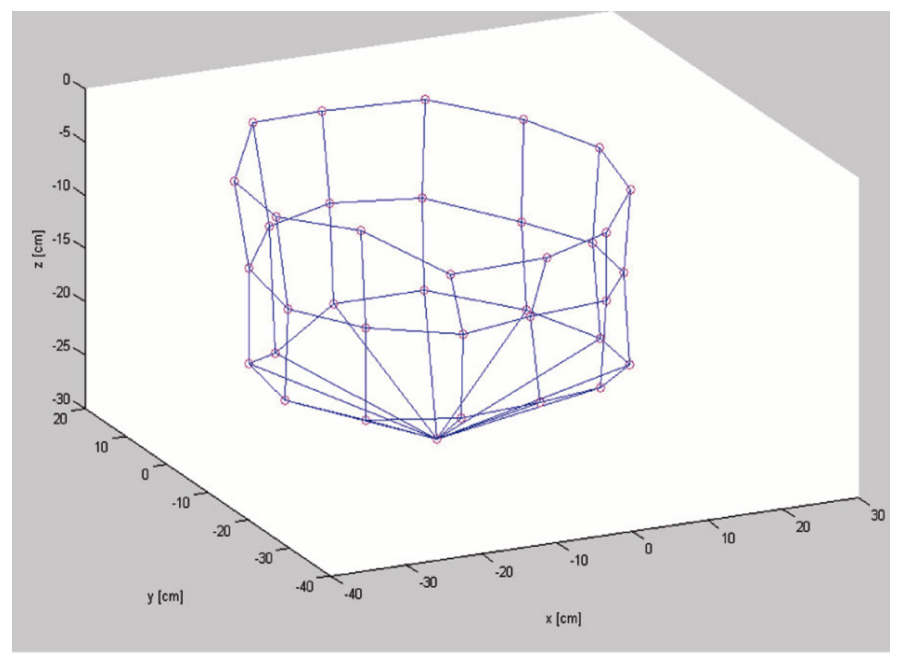

Fig. (9). Three-dimensional deformation of the net cage exposed to different current velocities from analysis of the stereo imaging method.

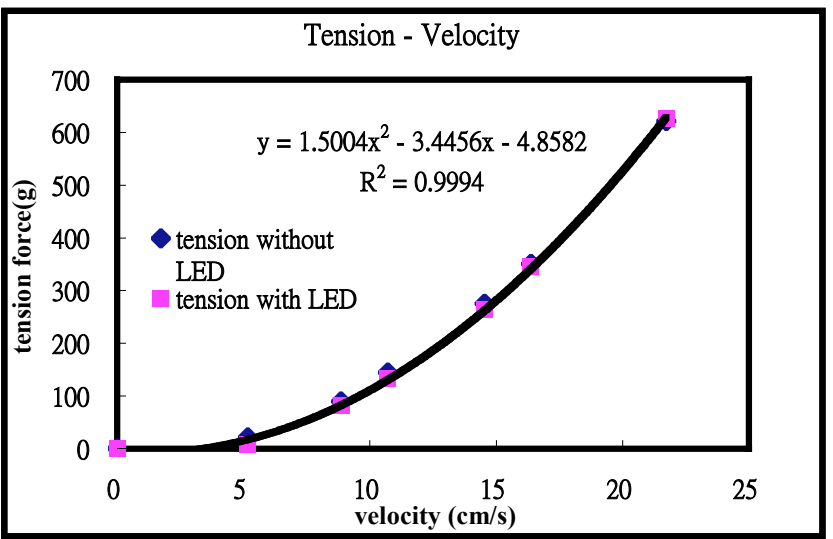

Fig. (8). Relationship between tension force of net cage and current velocity with /without LEDs marker.

used to measure the motion angle and the collar force by which the net cage was stirred in the currents. In evaluating the dynamic effects after the net cage was mounted with 37 LED markers, Fig. (8) shows that the tension forces of the mooring system for the cases of different velocities are almost the same with or without LED markers. From our investigation, the geometries of the net cage under different current velocities were the same for added LEDs or no LED markers. This proves that the light weight of the LED markers does not affect the motion of the net cage under current velocity. In addition, 37 LED markers were used to measure the internal volume of the net cage in this study. The accuracy of this method averaged about $90 \%$. Therefore, a non-intrusive experimental investigation of motion and geometry of the net cage in uniform flow is validated to be feasible. Furthermore,

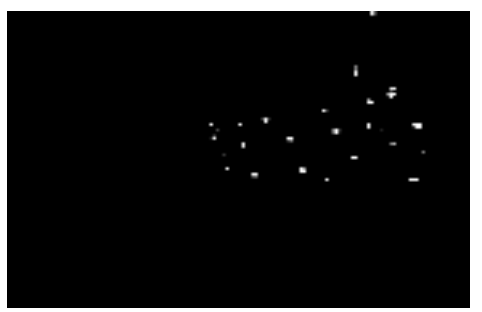

(b) $\mathrm{V}=22(\mathrm{~cm} / \mathrm{s})$

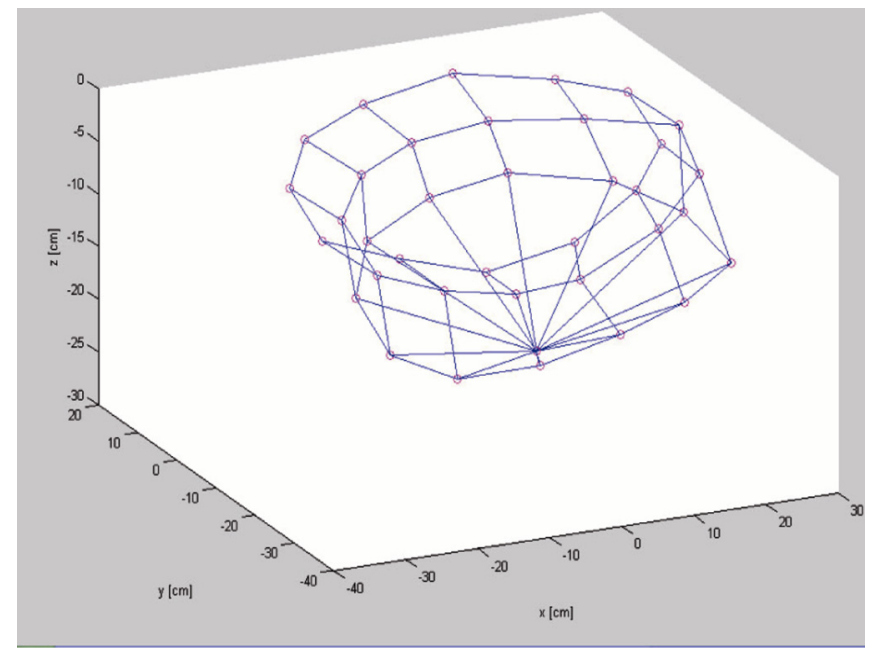

Fig. (9). Three-dimensional deformation of the net cage exposed to different current velocities from analysis of the stereo imaging method. 
three-dimensional deformation of the net cage for different current velocities can also be obtained from this stereo method. Fig. (9) shows the results of two cases for $V=6 \mathrm{~cm} / \mathrm{s}$ and $V=22 \mathrm{~cm} / \mathrm{s}$. Based on this analysis, the aquaculture application can be further understood in view of how the volume is reduced due to current exposure.

\section{Discussion of Hoop Motion and Tension Force}

Before considering the relationship between deformation and tension, the motion of the collar, tension force, and depressed volume for increasing current velocity must first be discussed. With the increase in current, the collar will gradually incline to below the free surface, increasing the tension force, and the volume of the net cage will be compressed and distorted. While the current velocity is under $6 \mathrm{~cm} / \mathrm{s}$, the collar is maintained on the free surface, and the inclination angle (the collar on the free surface is $0^{\circ}$ ) does not change.

However, the volume of the net cage quickly decreases in response to increasing tension force on the mooring system when the incline angle increases with added current velocity. Once the volume of the net cage is decreased, the collar then falls below the free surface, and the living space for fish will be constricted. This result is shown in Fig. (10).

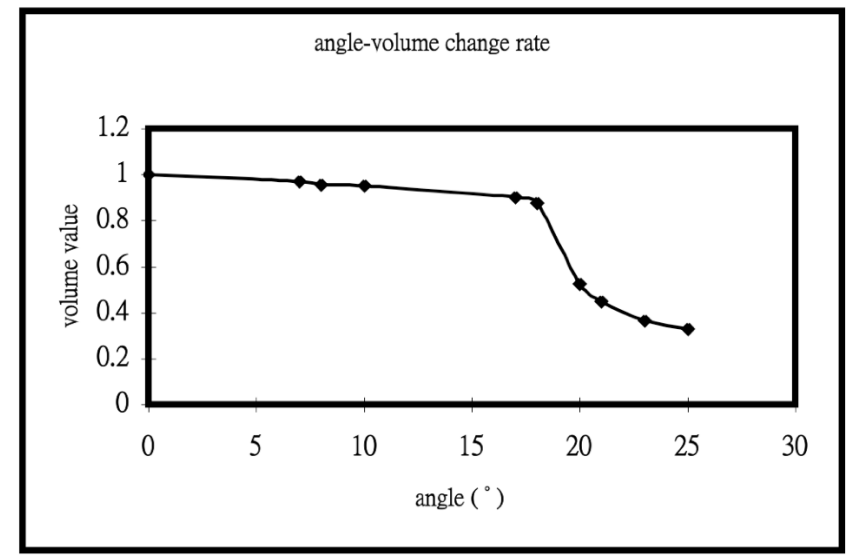

Fig. (10). Relationship between the change of angle with collar and the projected volume of net.

The phenomenon of the flow flux through the net cage and tension force is also interesting. The collar moves lower and lower below the free surface, while the tension force gets larger and larger with the increasing current velocity. Because of the increasing force, the projected net area (through which the current flows) is compressed with the additional inclined angle. While the projected area of the net is reduced, the flow discharge through the net is also decreased. Therefore, the relationship between the decreased discharge and tension force is as shown in Fig. (11). On the lateral side, the net will be constricted due to the current velocity passing through and the tension force of the mooring system on the hoop, which leads to a decrease in discharge through the net and an increase in the inclined angle.

\section{Analysis of Tension Force and Deformation of the Net Cage}

The volume deformation of the net cage as it is exposed to current is qualitatively described in Fig. (12). From these

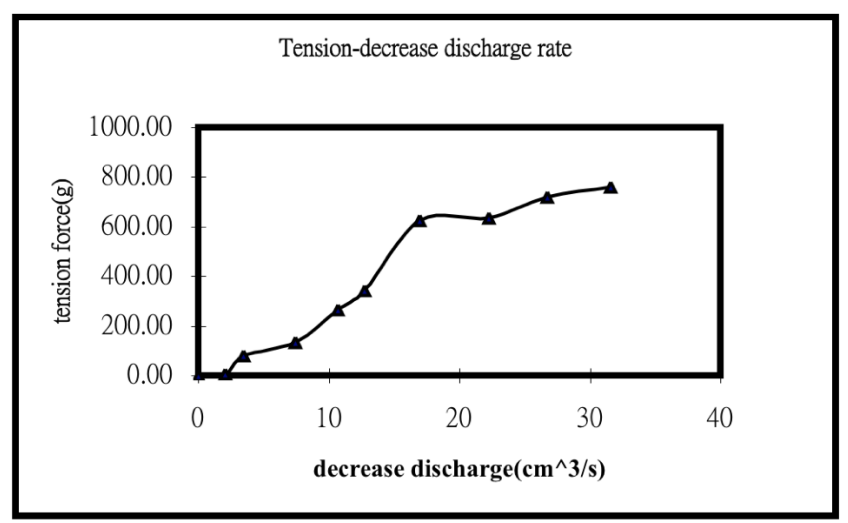

Fig. (11). Comparison of tension force and decreased discharge with the net cage.

images, it is clear that the deformation increases with increasing current velocity. Both the volume and exposed area reduction show the same qualitative features. For low velocities (12 $\mathrm{cm} / \mathrm{s})$, the deformation is moderate $(<5 \%)$, but for higher velocities, the deformation becomes substantially larger. When the current velocity exceeds $20 \mathrm{~cm} / \mathrm{s}$, the deformation rate of the net cage will be near $20 \%$. Huang [18] suggests that the deformation rate of the net cage should be kept below $40 \%$ for sufficient living space of fish. This configuration is suitable for the designation of the net cage under the range of current velocities. As we know, if the net cage is exposed to hydrodynamic force, the internal volume of the net cage will be changed. Meanwhile, a flux force will be produced when current flows through the net cage. In order to maintain the shape of the net cage with decreasing volume deformation, the bridle will generate a tension force to resist it. When current velocity is small, the tension force and deformation rate are small as well. This means the tension force is related to the volume change. The effect of the current operating on the net cage will show in the tension forces and the change of the shape of the net cage. Comparison between the tension forces and the value of $A_{e}{ }^{*} v^{2}$ is shown in Fig. (13). The result shows the relationship is almost linear between the tension forces and the change of value for $A_{e}{ }^{*} v^{2}$. Furthermore, when the current velocity is over $22 \mathrm{~cm} / \mathrm{s}$, the tension force will be increased quickly, and the net cage will be seriously deformed to the point where some of the LEDs will not be caught by two cameras. Using three cameras, as in this study, could aid in correctly determining the location of an LED in this situation.

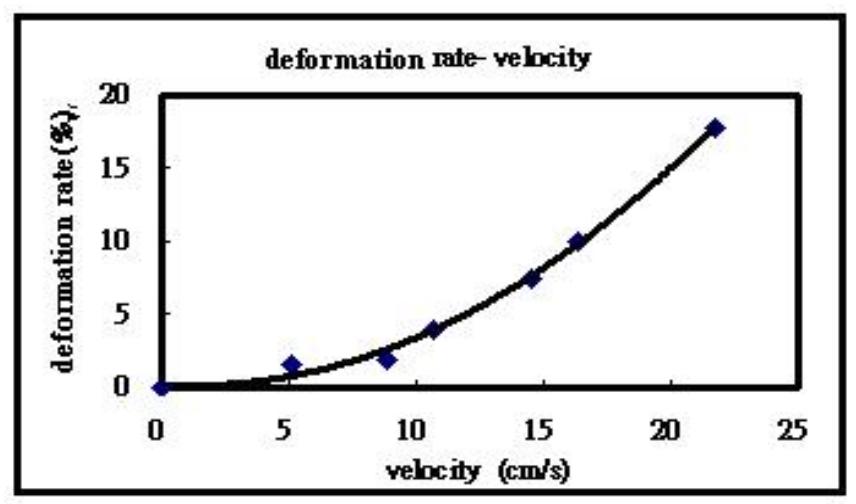

Fig. (12). Volume deformation rate for the net structure as a function of current velocity. 


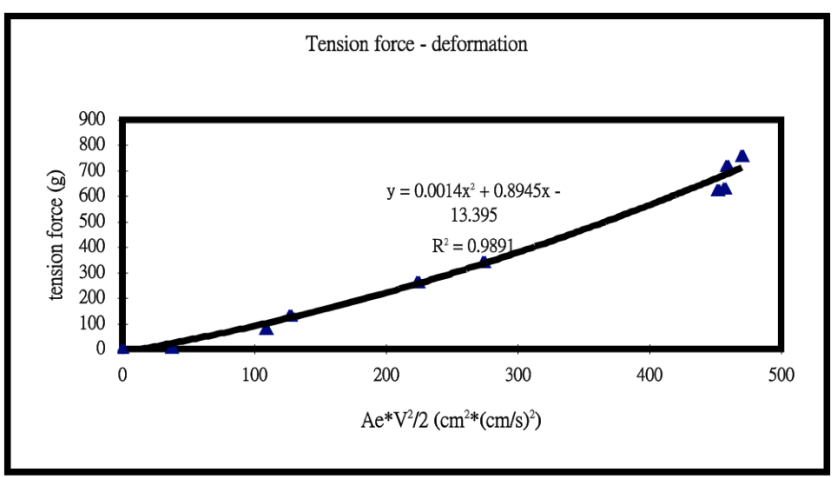

Fig. (13). Relation of the tension force and $A_{e} * v^{2}$ as the current flows through the net cage.

\section{CONCLUSIONS}

Non-intrusive stereo imaging measurement of threedimensional net geometry was established by tracing the positions of LED markers on the net in laboratory experiments.

Adding the LED did not significantly increase the weight on the net, helped to deduce the motion of net cage system, and also aided in the study of the physical phenomenon of the net cage. The 3D imaging method supplies more specific information necessary to consider more complicated flows, and obviously catches the motion of the net cage to obtain the closed experimental results. In $3 \mathrm{D}$ theory, the semi-symmetric situation is similar to the $2 \mathrm{D}$ field. Therefore, the $3 \mathrm{D}$ imaging technique provides a widely useful application. Use of three cameras could provide a backup to catch marks of LEDs that would be missed by two cameras in assessing cage behavior under larger current velocities, and therefore, the result could provide a closed solution for the experimental measurement.

More detailed information about deformation in the investigation of a flexible net structure can be obtained from this study. The experimental results suggest that the tension force and deformation are highly dependent on each other. The hydrodynamic force also appears to impact the stability and resistance of the net cage and collar. Understanding the dominating processes that govern fish cage and mooring system dynamics will become increasingly important as the aquaculture industry expands into more exposed locations. Therefore, measurements of the three-dimensional geometry (or motion) of a net cage exposed to hydrodynamic force in field experiments will be developed in the near future. With this, further in situ understanding and the use of validated analysis techniques, future net cage systems can potentially be designed to better withstand the rigors of the open ocean. If the risk can be reduced, the success of this new marine industry worldwide is more likely.

\section{ACKNOWLEDGEMENTS}

Funding for this research was provided by the NCKUNSYSU Research Center of Ocean Environment and Technology through the research program "Improvement Study on The Structure of Marine Net Cages" The authors would like to express profound thanks for the financial support of Ministry of Education, Taiwan, on this project.

\section{REFERENCES}

[1] J.S. Bessonneau, and D. Marichal, "Study of the dynamics of submerged supple sets (applications to trawls)", Ocean Eng., vol. 25, pp. 563-83, 1998.

[2] H.H. Lee, and S.W. Wang, "Analytical solution on the surge motion of tension leg twin-platform structural system", IEEE J. Ocean. Eng., vol. 30, pp. 59-78, 2005.

[3] D.B. Colbourne, and J.H. Allen, "Observations on motions and loads in aquaculture cages from full scale and model scale measurements", Aquacult. Eng., vol. 124, pp. 129-48, 2001.

[4] D.W. Fredriksson, "Open ocean fish cage abd mooring system dynamics", Ph.D. Dissertation. The University of New Hampshire, Durham, NH 2001.

[5] J.V. Aarsnes, H. Rudi, and G. Loland, Engineering for Offshore Fish Farming, Thomas Telford, London, 1990, pp. 137-152.

[6] H.H. Lee, and S.W. Wang, "Analytical solution on the surge motion of tension leg twin-platform structural system", Ocean Eng. Int. J., vol. 27, pp. 393-415, 2000.

[7] S.J. Tsorng, H. Capart, J.S. Lai, and D.L. Lai Young, "Threedimensional tracking of the long time trajectories of suspended particles in a lid-driven cavity flow", Exp. Fluids, vol. 40, pp. 314-28, 2006.

[8] M.J. Palczynski. "Fish cage physical modeling", M.Sc. Thesis. University of New Hampshire, Durham, NH 2000.

[9] B. Spinewine, H. Capart, M. Larcher, and Y. Zech, "Threedimensional Voronoï imaging methods for the measurement of nearwall particulate flows", Exp. Fluids, vol. 34, pp. 227-41, 2003.

[10] D. Douxchamps, D. Devriendt, H. Capart, C. Craeye, B. Macq, and Y. Zech, "Particle-based imaging method for the characterisation of complex fluid flows" Proc of the IEEE Oceans Conf., 2002.

[11] D. Douxchamps, D. Devriendt, H. Capart, C. Craeye, B. Macq, and Y. Zech, "Stereoscopic and velocimetric reconstructions of the free surface topography of antidune flows", Exp. Fluids, vol. 39, pp. 533$51,2005$.

[12] R.Y. Tasi, "A versatile camera calibration techniques for highaccuracy 3D machine vision metrology using off-the-shelf TV cameras and lenses", IEEE J. Robotics Auto, vol. 3, pp. 323-44, 1987.

[13] R. Jain, R. Kastui, and B.G. Schunck, Machine vision, McGraw-Hill, New York, 1995.

[14] N. Ahuja, "Dot pattern processing using Voronoi neighborhoods", IEEE PAMI, vol. 4, pp. 336-43, 1982.

[15] A. Okabe, B. Boots, and K. Sugihara, Spatial tessellations concepts and applications of Voronoi diagrams, Wiley, Chichester, 1992.

[16] Y. Murai, and F. Matsumoto, "Three-dimensional measurement of void fraction in a bubble plume using statistic stereoscopic image processing", Exp. Fluids, vol. 30, pp. 11-21, 2001.

[17] M. Klar, M. Jehle, B. Jähne, M. Detert, G.H. Jirka, H.-J. Köhler, and T. Wenka, "Simultaneous 3-D PTV and micro-pressure sensor equipment for flow analysis in a subsurface gravel layer. Proceedings of the 2nd International Conference", on Fluvial Hydraulics (M. Greco, A. Carravetta, and R. Della Morte, Eds.), Napoli, Italy (September 2002), 2004, pp. 703-712.

[18] C.C. Huang, and H.J. Tang, "Portable cage net study", Proc. $26^{\text {th }}$ Ocean Eng. Conf. In Taiwan, R.O.C., National Taiwan University, 2004, pp. 524-31. 